\title{
Subepithelial Vocal Fold Cyst: A Pearl on a String?
}

\author{
Nupur Kapoor Nerurkar, Sunita Chhapola Shukla
}

\begin{abstract}
Objective: Subepithelial vocal fold cyst (SEVFC) is an epithelium lined Sac within the lamina propria. These cysts are of two histologic types: Mucous retention cyst and epidermoid cyst. Rupture of the cystduring surgical excision and incomplete cyst wall removal with consequent recurrence of the cyst is not an infrequent problem. While performing cold-steel blunt microflap surgery for SEVFC, the first author observed thick fibrotic bands at the 12 and 6 o'clock position in all the cases, giving the appearance of a 'pearl (cyst) on a string (fibrotic bands)'. In most cases of cyst rupture, it took place during dissection of these bands. The aim of our study was to see if there was any significant difference in rupture and recurrence rate by sharp versus blunt dissection of these fibrotic bands.
\end{abstract}

Study design: Retrospective nonrandomized cohort comparison.

Materials and methods: Fifty-nine subepithelial cysts were operated by microflap technique. The first 26 cysts were taken in alternation for blunt vs sharp dissection of the fibrotic bands. As the results of sharp dissection were better, the subsequent 33 cysts were operated with sharp dissection of the anterior and posterior bands.

Results: Sharp dissection of the anterior and posterior bands during microflap dissection reduced the incidence of cyst rupture $(p=0.006)$ and recurrence $(p=0.007)$ of cyst formation.

Conclusion: SEVFC are anchored by thick fibrotic bands at the 12 and 6 o'clock position giving it the appearance of a 'pearl (cyst) on a string (fibrotic bands)'. Sharp dissection of these bands during microflap dissection reduces the incidence of cyst rupture and recurrence.

Keywords: Subepithelial vocal fold cyst, Mucous retention cyst, Epidermoid cyst, Microflap dissection, Cyst rupture, Cyst recurrence.

How to cite this article: Nerurkar NK, Shukla SC. Subepithelial Vocal Fold Cyst: A Pearl on a String? Int J Phonosurg and Laryngol 2012;2(2):53-56.

\section{Source of support: Nil}

\section{Conflict of interest: None declared}

\section{INTRODUCTION}

Subepithelial vocal fold cysts (SEVFC) were first described in the 19th century as epithelium-lined sacs within the lamina propria. They exist in the superficial lamina propria (SLP) and may be attached to the overlying epithelium or the underlying vocal ligament. ${ }^{1}$

Histologically, two types of cysts are found, mucous retention cysts and epidermoid cysts. M ucous retention cysts are formed due to an obstruction of the mucous gland duct, resulting in retained glandular secretions. These may occur spontaneously, during upper respiratory tract infection or with poor vocal hygiene and vocal abuse. They are often translucent and are lined with cuboidal or columnar epithelium (Fig. 1).

Epidermoid cysts are thought to develop from congenital cell rests in the subepithelium of the fourth and sixth branchial arches or from healing injured mucosa burying epithelium. They contain epithelium or accumulated keratin giving them a white color and are lined by stratified squamous epithelium (Fig. 2). Both types of cysts appear as oval masses beneath the epithelium. M ucous retention cyst may increase or decrease in size but epidermoid cyst rarely do so. ${ }^{1} \mathrm{~A}$ s the cyst enlarges, it increasingly interferes with the mucosal wave. SEVFC are usually found in the SLP and may occasionally be intracordal, i.e. within the vocal ligament. A focal thickening may also form as a reaction to trauma caused by the cyst on the contralateral

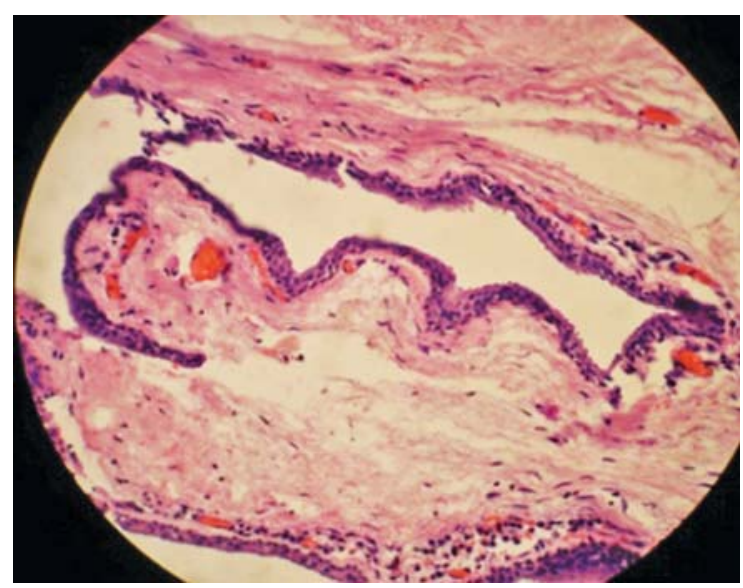

Fig. 1: Histopathology of mucous retention cyst

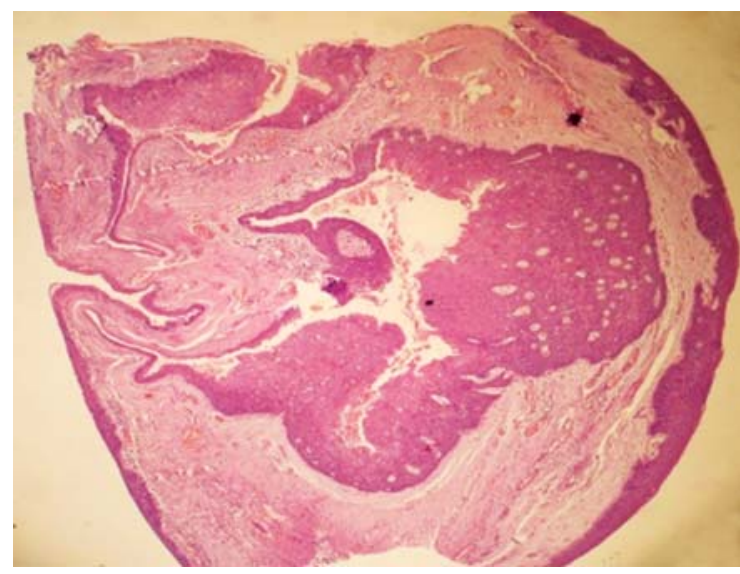

Fig. 2: Histopathology of epidermoid cyst 
vocal fold. Occasionally, bilateral cysts resembling vocal nodules may confound the diagnosis.

A new histological classification is proposed by Shvero et al. ${ }^{2} \mathrm{~A}$ : Cysts lined by columnar epithelium with mucous content; B: L ined by columnar epithelium with cilia; C: Lined by squamous epithelium without keratinization; $D$ : Lined by squamous epithelium with keratinization.

Surgeons may often be reluctant to perform surgery for SEVFC due to the technical difficulty in complete cyst resection, occasionally resulting in suboptimal vocal function or cyst recurrence. However, subepithelial infusion technique with microflap dissection helps in complete removal of the cyst wall and gives good results. ${ }^{1}$ The aim of our study was to see if there was any significant difference in rupture and recurrence rate of the SEVFC by sharp or blunt dissection of the fibrotic bands found anteriorly and posteriorly anchoring the cyst.

\section{MATERIALS AND METHODS}

Our study was performed on 59 subepithelial cysts operated by microflap technique in 56 patients in a 5-year period from June 2007 to J une 2012. All the surgeries were performed under general anesthesia. A subepithelial infusion was performed with 1 to 2 cc of $1 ; 10,000$ saline adrenaline to facilitate hydrodissection and maximally preserve the superficial lamina propria. An incision was taken as near the lateral edge of the cyst using a sickle knife or the acublade $\mathrm{CO}_{2}$ laser. Blunt dissection of the cyst was performed separating it from the overlying epithelium and the underlying lamina propria. During dissection of the cyst, fibrotic bands were found at the $60^{\prime}$ clock and $120^{\prime}$ clock position, giving the appearance of a 'pearl (cyst) on a string (fibrotic bands)' (Fig. 3). Blunt dissection of these fibrotic bands was performed in 13 cysts (Fig. 4) and sharp dissection of these fibrotic bands using scissors or A cuB lade $\mathrm{CO}_{2}$ laser (Fig. 5) in 46 cysts. If the cyst wall ruptured, the

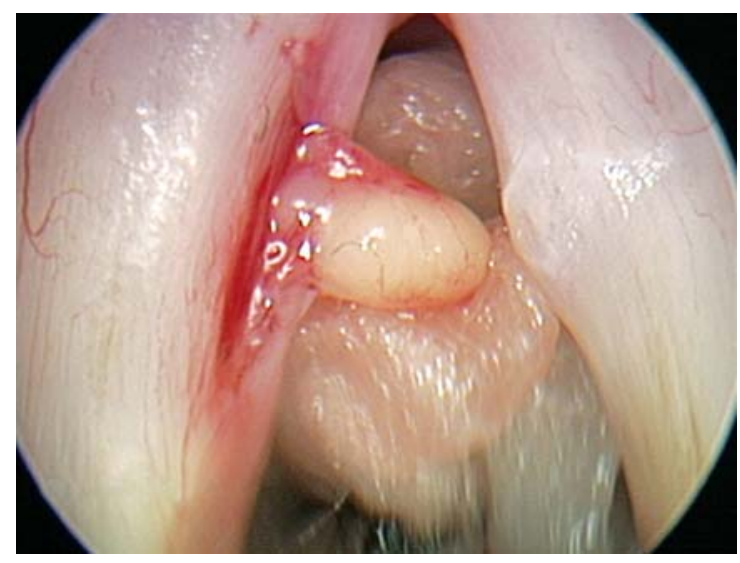

Fig. 3: Subepithelial epidermoid cyst dissected to demonstrate anterior and posterior fibrotic attachments giving an appearance of 'pearl on a string'

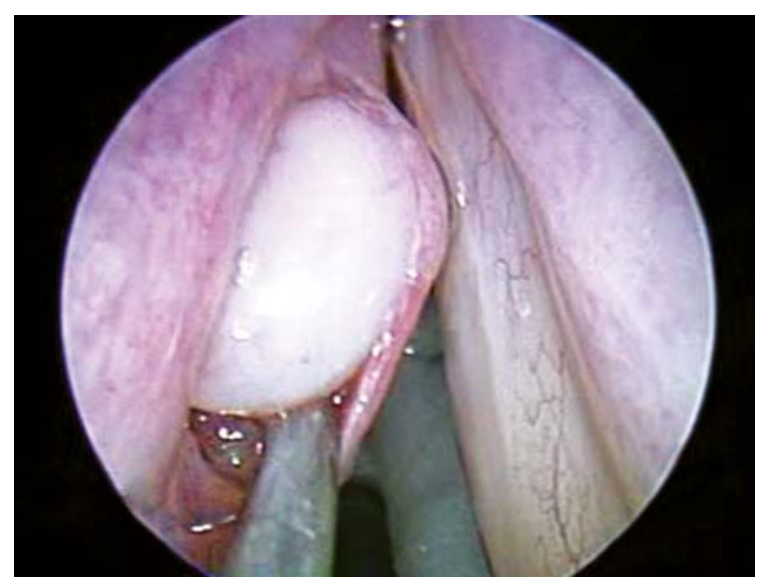

Fig. 4: Blunt dissection of posterior fibrotic band of an epidermoid cyst

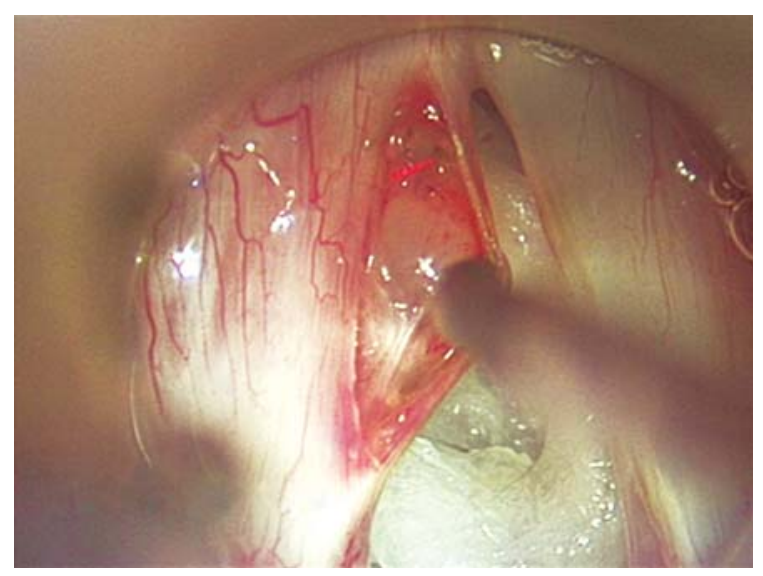

Fig. 5: Sharp dissection of anterior fibrotic and using AcuBlade $\mathrm{CO}_{2}$ laser in a mucous retention cyst

capsule was dissected from the SLP as completely as possible. Postoperatively, all patients were advised voice rest for 1 week followed by gradual restoration of vocal function aided with speech therapy.

\section{RESULTS}

Out of the 13 cysts that were dissected by blunt dissection, six cysts ruptured during dissection of the fibrotic bands. Furthermore, an extremely thin cyst wall led to rupture of another three cysts. Thus, total cysts ruptured were nine $(69.2 \%)$. In six of these nine cases, the cyst wall was felt to have been dissected out in toto. Recurrence of the cyst was observed in four patients (30.7\%). Histopathology revealed six mucous retention cysts and four epidermoid cysts. In three cysts the material sent was not sufficient for histopathology.

Out of the 46 cysts that were dissected using the technique of sharp dissection of the fibrotic bands, 12 cysts ruptured $(26.08 \%)$ due to a thin cyst wall $[p=0.006, O R=$ $6.38(95 \% \mathrm{Cl}: 1.65-24.6)]$. In 10 of these ruptured cysts the cyst wall could be dissected out completely. Recurrence 
was seen in one patient $(2.17 \%)$ [ $p=0.007, O R=20.0$ (95\% Cl: 1.995-200.5)]. Histopathology reveal ed 25 mucous retention cysts and 21 epidermoid cysts.

\section{DISCUSSION}

The vibratory surface of the human vocal fold is a complex structure. Repeated trauma from voice abuse leads to basement membrane disruption and thickening. This with poor vascular changes leads to the development of benign lesions that arise primarily within the lamina propria of the vocal fold. ${ }^{3}$ The delicate arrangement of the extracellular matrix proteins within the lamina propria permits passive movement of the epithelium, over the body, resulting in the formation of the mucosal wave as air is passed through the glottis as a release of building subglottic pressure. Most benign lesions occur in the superficial layer of the lamina propria, superficial to the vocal ligament and the thyroarytenoid muscle.

Patients of SEVFC present with hoarseness, increased effort of vocalization, vocal fatigue, pain while talking and soreness with voice use. The patient's voice is often diplophonic with pitch instability and splitting of fundamental frequency overtones. M aladaptive compensation patterns may lead to secondary muscle tension dysphonia. ${ }^{4}$

On indirect laryngoscopy, a cyst may appear as fullness in the vocal fold or as a lucent outline visible under the mucosal cover. A $n$ intracordal cyst should be suspected in a dysphonic patient when no obvious lesion is found on indirect laryngoscopy. On videostrobolaryngoscopy, the vocal folds appear asymmetric with an occasional evidence of a subepithelial mass. The mucosal wave overlying the cyst is decreased or absent in $100 \%$ of vocal cysts..$^{5-7}$ This is because the cyst tethers the mucosa, altering the biomechanical properties of the lamina propria and preventing mucosal wave propagation. ${ }^{4}$

Treatment requires a multidisciplinary approach regarding factors that contribute to voice disorders. M edical management aims at eliminating irritants and inflammatory conditions such as allergy and reflux laryngitis and maintaining good vocal hygiene. Speech therapy reduces abusive behaviors, improves vocal efficiency and modifies vocal habits. A though nodules and polyps may respond to conservative management, vocal cysts typically do not.

Surgical dissection of the intracordal cyst is very challenging as even minor changes in vocal fold structure can lead to suboptimal voice function, especially in professional voice users. Complete cyst removal with maximum preservation of superficial lamina propria enhances the vocal outcome. This is also facilitated by preincision hydrodissection in the subepithelial plane..$^{8-10}$ In addition, the deep layers of the lamina propria harbor fibroblasts that produce extracellular proteins. All effort is made to avoid dissection in this layer so as to prevent scarring along the vocal ligament and consequent tethering of the mucosal cover. ${ }^{11}$

In our study, we found thick anterior and posterior fibrotic bands tethering the SEVFC. Cutting these bands facilitated complete removal of the cyst with decreased incidence of rupture of the cyst. Similar inflammatory bands were also noted by Burns et $\mathrm{al}^{1}$ and Rosen et al. ${ }^{12}$

The outcome in our study was compared between the two groups using $\mathrm{M}$ antel-Haenszel $\mathrm{Chi}$-square test. Sharp dissection technique lead to decreased rupture of the cyst which is statistically significant $(p=0.006)$. The rate of recurrence also decreased with sharp dissection and was statistically significant $(p=0.007)$.

\section{CONCLUSION}

Our study implies that SEVFC have anterior and posterior fibrotic bands at 12 and $60^{\prime}$ clock position giving it a 'pearl on the string' appearance. Though most surgeons do combine both sharp and blunt dissection while excising subepithelial cysts, we recommend always looking for the anterior and posterior fibrotic bands anchoring the cyst and cutting these with sharp instruments in order to decrease the incidence of cyst rupture and recurrence.

\section{REFERENCES}

1. Burns ], et al. Phonomicrosurgical treatment of Intracordal vocal fold cysts in singers. Laryngoscope $2009 \mathrm{Feb} ; 119: 419-22$.

2. Shvero J, et al. Clinicopathologic study and classification of vocal cord cysts. Pathol Res Pract 2000;196(2):95-98.

3. Courey M S, Shohet JA, Scott M A, et al. Immunohistochemical characterization of benign laryngeal lesions. A nn Otol Rhinol L aryngol 1996 J ul;105(7):525-31.

4. Sataloff RT. The professional voice. In: Cummings CW, Fredrickson J M, Haker LA, et al (Eds). O tolaryngology: Head and Neck Surgery. St. Louis: M osby; 1986;3:2029-56.

5. Shohet JA, et al. V alue of videostroboscopic parameters in differentiating true vocal fold cysts from polyps. Laryngoscope 1996 J an;106(1Pt 1):19-26.

6. Sataloff RT, Spiegel JR, Hawkshaw MJ. Strobovideolaryngoscopy: Results and clinical value. Ann Otol Rhinol L aryngol 1991;100:725-27.

7. B ouchayer M, Cornut G, W itzig E, et al. E pidermoid cysts, sulci and mucosal bridges of the true vocal cord: A report of 157 cases. L aryngoscope 1985;95:1087-94.

8. Zeitels SM. Cysts. In: A tlas of phonomicrosurgery and other endolaryngeal procedures for benign and malignant disease. San Diego, CA: Singular; 2001:81-94. 
9. Kass ES, Hillman RE, Zeitels SM. The submucosal infusion technique in phonomicrosurgery. A nn Otol Rhinol Laryngol 1996;105:341-47.

10. Zeitels SM, Vaughan CW. A submucosal vocal fold infusion needle. Otolaryngol Head Neck Surgery 1991;105:478-79.

11. Sataloff RT, Spiegel JR, Heuer RJ, et al. Laryngeal minimicroflap: A new technique and reassessment of the microflap saga. J V oice 1995 J un; 9(2):198-204.

12. Rosen CA, A min M R, Sulica L, Simpson CB, M erati AL, Courey $M S$, et al. A dvances in Office-based diagnosis and treatment in laryngology. Invited paper. L aryngoscope 2009 N ov;119 Suppl 2:S185-212.

\section{ABOUT THE AUTHORS \\ Nupur Kapoor Nerurkar}

Consultant Laryngologist, Department of ENT, Bombay Hospital M umbai, M aharashtra, India

Correspondence Address: D-603, Simla H ouse, Napean Sea Road M umbai-400026, M aharashtra, India, e-mail: nupurkapoor@yahoo.com

\section{Sunita Chhapola Shukla}

Clinical A ssistant, Department of ENT, B ombay Hospital, M umbai Maharashtra, India 